\title{
On the apsidal motion of BP Vulpeculae
}

\author{
Csizmadia, Sz. ${ }^{\text {a }}$ Illés-Almár, E. ${ }^{\mathrm{b}}$, Borkovits, T. ${ }^{\mathrm{c}}$ \\ ${ }^{a}$ Institute of Planetary Research, German Aerospace Center, D-12489 Berlin, \\ Rutherfordstrasse 2., Germany \\ ${ }^{b}$ Konkoly Observatory, H-1525 Budapest, P. O. Box 67., Hungary \\ ${ }^{c}$ Baja Astronomical Observatory, H-6500 Baja, Szegedi út Kt. 766, Hungary
}

\begin{abstract}
BP Vulpeculae is a bright eclipsing binary system showing apsidal motion. It was found in an earlier study that it shows retrograde apsidal motion which contradicts theory. In this paper we present the first $B V$ light curve of the system and its light curve solution as well as seven new times of the minima from the years 1959-1963. This way we could expanded the baseline of the investigation to five decades. Based on this longer baseline we concluded that the apsidal motion is prograde agreeing with the theoretical expectations and its period is about 365 years and the determined internal structure constant is close to the theoretically expected one.
\end{abstract}

Key words: stars: binaries: eclipsing PACS: 97.80.Hn, Eclipsing binaries

\section{Introduction}

The eclipsing nature of the $10^{\text {th }}$ magnitude star BP Vulpeculae was discovered by Illés-Almár (1960) and she gave a period value of 1.938 days which was slightly corrected by Huth (1965). Then the star was neglected until the end of the 20th century when Lacv (1992) published $U B V$ colours of the system at certain phases. Later Lacy et al. (2003) presented more than 5000 data points in one colour $(V)$ and they have solved their light curve and determined very accurately absolute dimensions of the system. This $V$ light curve was again solved by a simple, but automatized code (Devor, 2005) and the result was similar to that of Lacy et al. (2003).

The system has an age of about 1 Gyr consisting of A7m V + F2m V spectral type components and is slightly eccentric $(e=0.0345$, Lacy et al., 2003). In eccentric binary star systems the apsidal line is revolving which is called apsidal motion and therefore the time difference between a primary minimum and the subsequent secondary minimum is variable. The apsidal motion is usually

Email addresses: szilard.csizmadia@dlr.de (Csizmadia, Sz.) 
caused by two effects: the tidal forces of the components and the effects of general relativity. In the case of a more or less ordinary eccentric binary, i.e. no very close third companion in the system, and no extreme stellar rotation, both phenomena predict that the apside shows a prograde motion. The total apsidal motion is a sum of the classical and relativistic contributions. It is worthy to mention that there are several systems, like DI Herculis or AS Camelopardalis where the observed apsidal motions highly differ from the theoretically predicted ones (see e.g. Claret, 1998, and references therein). It seems that the mentioned other effects, like third bodies etc. cause these pecularities (for an overview see Borkovits et al., 2007).

Regarding the case of BP Vul, Lacy (2003) established a very short apsidal motion period $(77 \pm 22 \mathrm{yr})$ and they found that it is a retrograde one. If $\mathrm{BP} \mathrm{Vul}$ really would have had a retrograde apsidal motion it would be a new representative of the systems which confronts theory and requires further study because a retrograde apsidal motion is in contradiction with theory. But the observational window in the work of Lacy (2003) was about one decade only therefore this rough estimation for the period of apsidal motion should be refined. For this refinement we present $B V$ observations of $\mathrm{BP}$ Vul which were obtained 45-49 years ago and were not published until now.

\section{Observations}

The $B V$ data points of $\mathrm{BP}$ Vul published here were obtained by one of us (E. Illés-Almár) during the years 1959-1963. The observations were carried out by the $60 \mathrm{~cm}$ Newtonian-telescope of the Konkoly Observatory, which is located at Budapest and it was installed in 1926. The detector was an 1P21 RCA photoelectric tube and $B$ and $V$ filters were used. The observations were reduced via a standard way. These differential magnitude data (they had been measured to a comparison star and transformed into standard system) are published here (see Tables 1-2 which are available electronically via the SIMBAD homepag $€^{1}$ ). The $B$ and $V$ curves can be seen in Figure 1 .

Using the method of Kwee \& van Woerden (1956) from these observations we determined seven new times of minima which are reported in Table 3. All the minima times we used for the analysis can be found in that Table, too.

\section{Period analysis}

The $O-C$ values were calculated with the following ephemeris:

$$
\text { Min } \mathrm{I}=2436860.3311+1.9403494 \times E
$$

where the initial epoch was our first primary minimum observation while the period was taken from Lacy et al. (2003). The $O-C$ diagram is presented in

\footnotetext{
${ }^{1}$ http://simbad.u-strasbg.fr/simbad/
} 
Table 3: Observed times of minima of BP Vul. Meaning of weights (W): 10: $\mathrm{CCD} /$ photoelectric minimum, 2: plate minimum, 1: visual observation, 0 : not used for the calculation because it is an outlier.

\begin{tabular}{|c|c|c|c|c|c|c|c|}
\hline $\begin{array}{c}\text { Time of Min. } \\
\text { (HJD-2 } 400 \text { 000) }\end{array}$ & $\overline{\mathrm{W}}$ & Type & Ref. & $\begin{array}{c}\text { Time of Min. } \\
\text { (HJD-2 } 400000)\end{array}$ & $\overline{\mathrm{W}}$ & Type & Ref. \\
\hline 21787.723 & 2 & $\mathrm{p}$ & 1 & 37898.427 & 2 & $\mathrm{p}$ & 2 \\
\hline 22144.750 & 2 & $\mathrm{p}$ & 1 & 37933.365 & 2 & $\mathrm{p}$ & 2 \\
\hline 22637.569 & 2 & $\mathrm{p}$ & 1 & 37935.294 & 2 & $\mathrm{p}$ & 2 \\
\hline 23607.741 & 2 & $\mathrm{p}$ & 1 & 38001.256 & 2 & $\mathrm{p}$ & 2 \\
\hline 25831.362 & 2 & $\mathrm{p}$ & 2 & 38255.437 & 2 & $\mathrm{p}$ & 2 \\
\hline 26465.843 & 2 & $\mathrm{p}$ & 1 & 38288.425 & 10 & $\mathrm{p}$ & 3 \\
\hline 26512.439 & 2 & $\mathrm{p}$ & 2 & 38323.326 & 2 & $\mathrm{p}$ & 2 \\
\hline 26535.718 & 2 & $\mathrm{p}$ & 1 & 38614.433 & 2 & $\mathrm{p}$ & 2 \\
\hline 26545.469 & 2 & $\mathrm{p}$ & 2 & 38938.446 & 1 & $\mathrm{p}$ & 4 \\
\hline 26647.308 & 2 & s & 2 & 38938.452 & 1 & $\mathrm{p}$ & 4 \\
\hline 26648.332 & 2 & $\mathrm{p}$ & 2 & 38938.453 & 1 & $\mathrm{p}$ & 4 \\
\hline 26868.522 & 2 & $\mathrm{~s}$ & 2 & 41082.509 & 1 & $\mathrm{p}$ & 5 \\
\hline 26930.399 & 0 & $\mathrm{p}$ & 2 & 41107.549 & 0 & $\mathrm{p}$ & 5 \\
\hline 27965.784 & 2 & $\mathrm{p}$ & 1 & 41115.516 & 1 & $\mathrm{p}$ & 5 \\
\hline 28074.410 & 2 & $\mathrm{p}$ & 2 & 41965.387 & 1 & $\mathrm{p}$ & 6 \\
\hline 28078.299 & 2 & $\mathrm{p}$ & 2 & 42386.456 & 2 & $\mathrm{p}$ & 1 \\
\hline 28460.527 & 2 & $\mathrm{p}$ & 1 & 42666.772 & 2 & $\mathrm{~s}$ & 1 \\
\hline 29073.744 & 2 & $\mathrm{p}$ & 1 & 43317.780 & 2 & $\mathrm{p}$ & 1 \\
\hline 29114.476 & 2 & $\mathrm{p}$ & 2 & 43423.529 & 2 & $\mathrm{~s}$ & 1 \\
\hline 29857.637 & 2 & $\mathrm{p}$ & 1 & 44757.540 & 1 & $\mathrm{p}$ & 7 \\
\hline 31318.694 & 2 & $\mathrm{p}$ & 1 & 44875.875 & 2 & $\mathrm{p}$ & 1 \\
\hline 33854.692 & 2 & $\mathrm{p}$ & 1 & 45114.551 & 1 & $\mathrm{p}$ & 8 \\
\hline 34209.792 & 2 & $\mathrm{p}$ & 1 & 45504.566 & 1 & $\mathrm{p}$ & 9 \\
\hline 34221.446 & 2 & $\mathrm{p}$ & 2 & 45541.452 & 1 & $\mathrm{p}$ & 10 \\
\hline 34580.427 & 2 & $\mathrm{p}$ & 2 & 45611.295 & 1 & $\mathrm{p}$ & 11 \\
\hline 35224.585 & 2 & $\mathrm{p}$ & 2 & 45611.302 & 1 & $\mathrm{p}$ & 11 \\
\hline 35226.576 & 2 & $\mathrm{p}$ & 2 & 45636.490 & 2 & $\mathrm{p}$ & 1 \\
\hline 35721.355 & 2 & $\mathrm{p}$ & 2 & 45785.882 & 2 & $\mathrm{p}$ & 1 \\
\hline 36433.446 & 2 & $\mathrm{p}$ & 2 & 45855.778 & 2 & $\mathrm{p}$ & 1 \\
\hline 36790.450 & 2 & $\mathrm{p}$ & 2 & 45931.461 & 1 & $\mathrm{p}$ & 12 \\
\hline 36859.3445 & 10 & $\mathrm{~s}$ & 3 & 45933.404 & 1 & $\mathrm{p}$ & 13 \\
\hline 36860.331 & 10 & $\mathrm{p}$ & 2 & 46003.248 & 1 & $\mathrm{p}$ & 14 \\
\hline 36860.3311 & 10 & $\mathrm{p}$ & 3 & 46290.424 & 1 & $\mathrm{p}$ & 15 \\
\hline 37116.458 & 2 & $\mathrm{p}$ & 2 & 46321.464 & 1 & $\mathrm{p}$ & 15 \\
\hline 37438.547 & 2 & $\mathrm{p}$ & 2 & 46356.387 & 1 & $\mathrm{p}$ & 15 \\
\hline 37506.4677 & 10 & $\mathrm{p}$ & 3 & 46385.508 & 2 & $\mathrm{p}$ & 1 \\
\hline 37543.3344 & 10 & $\mathrm{p}$ & 3 & 46534.864 & 2 & $\mathrm{p}$ & 1 \\
\hline 37572.4389 & 10 & $\mathrm{p}$ & 3 & 46612.497 & 1 & $\mathrm{p}$ & 16 \\
\hline 37642.260 & 2 & $\mathrm{p}$ & 2 & 46612.497 & 1 & $\mathrm{p}$ & 16 \\
\hline 37867.3714 & 10 & $\mathrm{p}$ & 3 & 46612.500 & 1 & $\mathrm{p}$ & 16 \\
\hline
\end{tabular}

1: Torres \& Guilbault (2003); 2: Huth (1965); 3: Present paper; 4: BAV 7; 5: BBSAG 30; 6: BBSAG 12; 7: BAV 34; 8: BBSAG 61; BBSAG 67 10: BAV 38; 11: BBSAG 69; 12: BAAVSS 61; 13: BBSAG 73; 14: BBSAG 74; 15: BBSAG 78; 16: BRNO 28 
Table 3: (Continue.)

\begin{tabular}{|c|c|c|c|c|c|c|c|}
\hline $\begin{array}{c}\text { Time of Min. } \\
\text { (HJD-2 } 400 \text { 000) }\end{array}$ & $\overline{\mathrm{W}}$ & Type & Ref. & $\begin{array}{c}\text { Time of Min. } \\
\text { (HJD-2 } 400 \text { 000) }\end{array}$ & $\bar{W}$ & Type & $\overline{\text { Ref. }}$ \\
\hline 46612.501 & 1 & $\mathrm{p}$ & 16 & 49216.450 & 1 & p & 29 \\
\hline 46612.502 & 1 & $\mathrm{p}$ & 16 & 49216.461 & 1 & $\mathrm{p}$ & 29 \\
\hline 46612.503 & 1 & $\mathrm{p}$ & 16 & 49216.464 & 1 & $\mathrm{p}$ & 30 \\
\hline 46612.504 & 1 & $\mathrm{p}$ & 16 & 49216.468 & 1 & $\mathrm{p}$ & 30 \\
\hline 46612.506 & 1 & $\mathrm{p}$ & 16 & 49218.393 & 1 & $\mathrm{p}$ & 29 \\
\hline 46612.507 & 1 & $\mathrm{p}$ & 16 & 49218.402 & 1 & $\mathrm{p}$ & 29 \\
\hline 46612.509 & 1 & $\mathrm{p}$ & 16 & 49218.411 & 1 & $\mathrm{p}$ & 30 \\
\hline 46614.441 & 1 & $\mathrm{p}$ & 16 & 49218.413 & 1 & $\mathrm{p}$ & 31 \\
\hline 46614.445 & 1 & $\mathrm{p}$ & 16 & 49251.390 & 1 & $\mathrm{p}$ & 30 \\
\hline 46614.447 & 1 & $\mathrm{p}$ & 16 & 49321.242 & 1 & $\mathrm{p}$ & 30 \\
\hline 46614.447 & 1 & $\mathrm{p}$ & 16 & 49934.390 & 1 & $\mathrm{p}$ & 32 \\
\hline 46614.448 & 1 & $\mathrm{p}$ & 16 & 49967.380 & 1 & $\mathrm{p}$ & 32 \\
\hline 46614.452 & 1 & $\mathrm{p}$ & 16 & 50002.313 & 1 & $\mathrm{p}$ & 32 \\
\hline 46678.476 & 1 & $\mathrm{p}$ & 16 & 50324.400 & 1 & $\mathrm{p}$ & 33 \\
\hline 46678.478 & 1 & $\mathrm{p}$ & 16 & 50357.372 & 1 & $\mathrm{p}$ & 33 \\
\hline 46709.547 & 2 & $\mathrm{p}$ & 1 & 50547.551 & 1 & $\mathrm{p}$ & 34 \\
\hline 46973.428 & 1 & $\mathrm{p}$ & 17 & 50681.418 & 1 & $\mathrm{p}$ & 34 \\
\hline 47026.696 & 2 & $\mathrm{~s}$ & 1 & 50718.283 & 1 & $\mathrm{p}$ & 35 \\
\hline 47039.370 & 1 & $\mathrm{p}$ & 18 & 50751.277 & 1 & $\mathrm{p}$ & 35 \\
\hline 47064.618 & 2 & $\mathrm{p}$ & 1 & 51036.496 & 1 & $\mathrm{p}$ & 36 \\
\hline 47361.497 & 1 & $\mathrm{p}$ & 19 & 51063.6717 & 10 & $\mathrm{p}$ & 37 \\
\hline 47363.441 & 1 & $\mathrm{p}$ & 19 & 51128.645 & 10 & s & 37 \\
\hline 47392.530 & 1 & $\mathrm{p}$ & 20 & 51129.646 & 10 & $\mathrm{p}$ & 37 \\
\hline 47392.535 & 1 & $\mathrm{p}$ & 20 & 51327.564 & 1 & $\mathrm{p}$ & 38 \\
\hline 47392.543 & 1 & $\mathrm{p}$ & 20 & 51364.416 & 1 & $\mathrm{p}$ & 39 \\
\hline 47396.424 & 1 & $\mathrm{p}$ & 19 & 51397.4114 & 10 & $\mathrm{p}$ & 40 \\
\hline 47431.331 & 1 & $\mathrm{p}$ & 21 & 51464.3104 & 10 & s & 40 \\
\hline 47466.271 & 1 & $\mathrm{p}$ & 21 & 52031.90450 & 10 & $\mathrm{p}$ & 41 \\
\hline 47788.3674 & 10 & $\mathrm{p}$ & 22 & 52064.89086 & 10 & $\mathrm{p}$ & 41 \\
\hline 47790.313 & 1 & $\mathrm{p}$ & 23 & 52096.86757 & 10 & s & 41 \\
\hline 47823.313 & 1 & $\mathrm{p}$ & 24 & 52098.80834 & 10 & $\mathrm{~s}$ & 41 \\
\hline 48112.405 & 1 & $\mathrm{p}$ & 25 & 52099.8166 & 10 & $\mathrm{p}$ & 41 \\
\hline 48112.412 & 1 & $\mathrm{p}$ & 25 & 52101.75702 & 10 & $\mathrm{p}$ & 41 \\
\hline 48147.327 & 1 & $\mathrm{p}$ & 25 & 52164.7794 & 10 & $\mathrm{~s}$ & 41 \\
\hline 48176.432 & 1 & $\mathrm{p}$ & 25 & 52165.78900 & 10 & $\mathrm{p}$ & 41 \\
\hline 48533.457 & 1 & $\mathrm{p}$ & 26 & 52425.79570 & 10 & $\mathrm{p}$ & 42 \\
\hline 48723.609 & 1 & $\mathrm{p}$ & 27 & 52487.88765 & 10 & $\mathrm{p}$ & 42 \\
\hline 48859.422 & 1 & $\mathrm{p}$ & 28 & 52488.81917 & 10 & $\mathrm{~s}$ & 42 \\
\hline
\end{tabular}

17: BBSAG 84; 18: BBSAG 86; 19: BBSAG 89; 20: BRNO 30; 21: BBSAG 90; 22: BAV 56 23: BBSAG 92; 24: BBSAG 93; 25: BBSAG 96; 26: BBSAG 99; 27: BBSAG 101; 28: BBSAG 102 29: BRNO 31; 30: BBSAG 105; 31: BBSAG 104; 32: BBSAG 110; 33: BBSAG 113; 34: BBSAG 115; 35: BBSAG 116 36: BBSAG 118; 37: Lacv et al. (1999); 38: BBSAG 120; 39: BRNO 32; 40 Agerer et al. (2001); 41: Lacy et al. (2002); 42: Lacy (2002) 
Table 3: (Continue.)

\begin{tabular}{cccc|cccc}
\hline \hline $\begin{array}{c}\text { Time of Min. } \\
\text { (HJD-2 400 000) }\end{array}$ & W & Type & Ref. & $\begin{array}{c}\text { Time of Min. } \\
\text { (HJD-2 400 000) }\end{array}$ & W & Type & Ref. \\
\hline \hline 52495.64880 & 10 & $\mathrm{p}$ & 42 & 53526.9042 & 10 & $\mathrm{~s}$ & 47 \\
52562.5517 & 10 & $\mathrm{~s}$ & 42 & 53527.91289 & 10 & $\mathrm{p}$ & 47 \\
52595.5379 & 10 & $\mathrm{~s}$ & 42 & 53898.5192 & 10 & $\mathrm{p}$ & 48 \\
52724.589 & 1 & $\mathrm{p}$ & 43 & 53933.4432 & 10 & $\mathrm{p}$ & 49 \\
52782.8192 & 10 & $\mathrm{p}$ & 44 & 53987.7740 & 10 & $\mathrm{p}$ & 50 \\
52814.7964 & 10 & $\mathrm{~s}$ & 44 & 54026.5809 & 10 & $\mathrm{p}$ & 50 \\
52817.74512 & 10 & $\mathrm{p}$ & 44 & 54325.3939 & 10 & $\mathrm{p}$ & 51 \\
53169.8789 & 10 & $\mathrm{~s}$ & 45 & 54388.4173 & 10 & $\mathrm{~s}$ & 51 \\
53186.4111 & 10 & $\mathrm{p}$ & 46 & & & & \\
\hline
\end{tabular}

43: Diethelm (2003); 44: Lacy (2003); 45: Lacy (2004); 46: Zejda (2004); 47: Lacv (2006); 48: Hübscher et al. (2006); 49: Hübscher (2007); 50: Lacv (2007); 51: Hübscher et al. (2008)

Figure 2, It is clear from Figure 2 that the time lag between the primary and secondary minima has changed so the apsidal motion is clearly present. In the following calculations CCD and photoelectric times of minima had weights of 10, plate minima had 2, visual observations had 1 .

Using a second-order approximation in the eccentricity, the times of primary and secondary minima will occur at the times given below:

$$
\begin{gathered}
\text { Min } \mathrm{I}=T_{0}+E P_{\mathrm{s}}-\frac{e P_{\mathrm{a}}}{\pi} \cos \omega_{E}+\frac{3}{8} \frac{e^{2} P_{\mathrm{a}}}{\pi} \sin 2 \omega_{E}+\ldots \\
\text { Min II }=T_{0}+E P_{\mathrm{s}}-\frac{P_{\mathrm{a}}}{2}+\frac{e P_{\mathrm{a}}}{\pi} \cos \omega_{E}+\frac{3}{8} \frac{e^{2} P_{\mathrm{a}}}{\pi} \sin 2 \omega_{E}-\ldots
\end{gathered}
$$

where $T_{0}$ are the epoch of a primary minimum, $P_{\mathrm{a}}$ is the anomalistic period, $P_{\mathrm{s}}$ is the sidereal period, i.e. $P_{\mathrm{s}} \approx P_{\mathrm{a}}\left(1-\omega^{\prime} / 2 \pi\right)$, $e$ is the eccentricity, $\omega_{E}=\omega_{0}+\omega^{\prime} E$ where $\omega^{\prime}=2 \pi P / U$. $U$ is the apsidal motion period.

Applying these formulae we determined the apsidal motion period with the upgraded version of LiteAM software developed by T. Borkovits (see e.g. Borkovits et al., 2002). We found from the fitting of the $O-C$ curve that $U / P=$ $68700 \pm 500$ and this means $U=365$ years, $T_{0}=51063.6537 \pm 0.0001(H J D)$ and $\omega_{0}=150^{\circ} \pm 5^{\circ}$. This latter value is in good agreement with $\omega=154.7^{\circ} \pm 3.9^{\circ}$ found by spectroscopic measurements (Lacy et al., 2003). The apsidal motion period yields $\dot{\omega} \approx 1.0^{\circ} /$ year.

Note that the $O-C$ curve is not well-covered yet. There is need for more observations to determine an exact value of the apsidal motion period in BP Vul - our value given above can be regarded as a first approximation. But more interesting than the exact value of this period is that the new value yielded a prograde motion of the semi-major axis instead of a retrograde one. 


\section{Light curve solution}

For the light curve solution we used the Wilson-Devinney Code Wilson, 1998). The free parameters were the inclination, the dimensionless surface potentials, argument of periastron and its time-derivative and the luminosities of the components. Limb-darkeking coefficients were fixed and these fixed values were interpolated ones from tables of van Hamme (1993). Gravity darkening and reflexion coefficients were also fixed. Mass ratio, surface temperatures of the components and eccentricity of the orbit were fixed at the values given in Lacy et al. (2003). Then differential correction analysis were carried out and the stopping criteria was that the change in the parameters in the final step should be lower than its standard deviations. Since BP Vul has a fast apsidal motion (see previous Section) we used time as an independent variable during the modeling rather than phase. The result of the light curve solution can be found in Table 4

Comparing our results to the one of Lacy et al. (2003) we found a remarkably excellent agreement in luminosity ratio, but other elements are slightly different. However, the precision of our light curve does not reach the precision of their one although we have colour information, too. Moreover, they used the so-called EBOP code (Popper \& Etzel, 1981) which has a slightly different input physics. Since we solve these old light curves for the purpose to determine the argument of periastron independently, these slight differences do not destroy the validity of our light curve solution.

Thus we concentrate the position of the periastron hereafter. As one can see from Table $4 \omega=126^{\circ} \pm 5^{\circ}$ at epoch HJD $=2436860.3311$ (our adopted epoch) which nearly corresponds 1959 October 18 . From their spectroscopic measurements Lacy et al. (2003) had given $\omega=154.7^{\circ}$ for epoch HJD 2451023.254 which nearly corresponds to 1998 July 28 . It is easy to compute that these two measurements yield $0.74^{\circ} / \mathrm{yr}$ apsidal motion or $U=486 \pm 57$ years.

This value is more than the 365 years apsidal motion period - determined from the $O-C$ analysis - by about 120 years. According to us this $33 \%$ difference is not because the $O-C$ diagram is not well-covered and a new light curve solution based on multi-colour observations are needed.

However, the fitted $\dot{\omega}$ gives $1.0^{\circ} / \mathrm{yr}$ which is fully in agreement with the results of the $O-C$ analysis. Regarding the uncertainty in the position of $\omega$ determined from these old light curves, one can conclude that very likely the light curve solution gives $\dot{\omega} \approx 1.0^{\circ} / \mathrm{yr}$.

\section{The internal structure constant $k_{2}$}

Our next calculations are based on Gimenez (1985). From the known eccentricity, masses and period given in Lacy et al. (2003) one can calculate that the relativistic contribution to the apsidal motion in BP Vulpeculae is $7.529 \cdot 10^{-4}$ degree/cycle. From the observed $U=365$ years see above we found $\dot{\omega}_{\text {obs }}=$ $5.24 \cdot 10^{-3}$ degree/cycle. So the Newtonian term in the apsidal motion is $\dot{\omega}_{N}=\dot{\omega}_{\text {obs }}-\dot{\omega}_{\text {rel }}=4.49 \cdot 10^{-4}$ degree $/$ cycle. 
Table 4: Light curve solution of BP Vulpeculae. Denotions have their usual meaning. Mode 0 of the Wilson-Devinney Code was used (see Wilson, 1998). $r_{1,2}$ were derived from $\Omega_{1,2}$ and $q$ by the WD-code itself.

\begin{tabular}{cccc}
\hline \hline Quantity & & This paper & Lacv et al. (2003) \\
\hline$i$ & adjusted & $86.64 \pm 0.16$ & 87.67 \\
$L_{1} / L_{\text {tot }}(\mathrm{B})$ & adjusted & $0.719 \pm 0.02$ & - \\
$L_{1} / L_{\text {tot }}(\mathrm{V})$ & adjusted & $0.696 \pm 0.02$ & 0.718 \\
$\omega$ & adjusted & $126^{\circ} \pm 5^{\circ}$ & $154.7^{\circ}$ \\
$\dot{\omega}$ & adjusted & $1.006^{\circ} \pm 0.002^{\circ}$ & - \\
$\Omega_{1}$ & adjusted & $7.04 \pm 0.09$ & - \\
$\Omega_{2}$ & adjusted & $6.89 \pm 0.07$ & - \\
$H J D 0$ & fixed & 2436860.3311 & 2451023.254 \\
mean $r_{1}$ & derived & $0.162 \pm 0.027$ & 0.1931 \\
mean $r_{2}$ & derived & $0.141 \pm 0.031$ & 0.1552 \\
$g_{1}$ & fixed & 1.0 & - \\
$g_{2}$ & fixed & 1.0 & - \\
$A_{1}$ & fixed & 1.0 & - \\
$A_{2}$ & fixed & 1.0 & - \\
$T_{1}$ & fixed & $7709 \mathrm{~K}$ & $7709 \mathrm{~K}$ \\
$T_{2}$ & fixed & $6823 \mathrm{~K}$ & $6823 \mathrm{~K}$ \\
$x_{1, \text { bol }}$ & fixed & 0.538 & - \\
$x_{2, \text { bol }}$ & fixed & 0.467 & - \\
$x_{1, \mathrm{~B}}$ & fixed & 0.604 & - \\
$x_{2, \mathrm{~B}}$ & fixed & 0.621 & - \\
$x_{1, \mathrm{~V}}$ & fixed & 0.534 & $0.50 \pm 0.03$ \\
$x_{2, \mathrm{~V}}$ & fixed & 0.507 & $0.56 \pm 0.03$ \\
$e$ & fixed & 0.0345 & 0.0345 \\
$q$ & fixed & 0.811 & 0.811 \\
\hline \hline
\end{tabular}


Using the well-known relationship

$$
k_{2, \mathrm{obs}}=\frac{1}{c_{21}+c_{22}} \frac{\dot{\omega}_{N}}{360^{\circ}}
$$

we found $\log k_{2, \text { obs }}=-2.66 \pm 0.08$. Here $c_{21}$ and $c_{22}$ are the functions of eccentricity, mass ratio and fractional radii of the components and their precise form is given in Gimenez (1985).

Using the tables of Claret (2004) with $\mathrm{X}=0.70, \mathrm{Z}=0.02, \mathrm{t}=1 \mathrm{Gyr}$ and with mixing length parameter $\alpha=1.68$ and overshooting parameter $\alpha_{O V}=0.2$ we could calculate $\log k_{2, \text { theo }}=-2.47$. Regarding the uncertainties in the determined apsidal motion period which appears in the determination of $k_{2}$ internal structure constant we may conclude that the observed and theoretically expected values are close to each other. Also note that we have only one secondary minimum from the 1960s which is a key point in similar calculations.

\section{Summary}

BP Vulpeculae is an eccentric eclipsing binary star showing the so-called periastron-precession effect. Lacy et al. (2003) concluded that this effect causes a retrograde motion of the semi-major axis and it has a period of $77 \pm 22$ years based on their about one decade long observational material. However, retrograde motion contradicts theory. Their explanation was that a possible third body in the system could perturb the orbit yielding the observed peculiar periastron precession. Nevertheless no spectroscopic evidence was found by them for such a third body.

BP Vul was observed at the Konkoly Observatory more than forty years before the work of Lacy et al. (2003) by one of the authors of this study. This observational material allowed us to determine the times of six primary and one secondary minima. With these early observations the baseline could be expanded to approximately five decades which was enough to refine the apsidal motion period determined by Lacy et al. (2003). Our $O-C$ analysis based on the extended time-line showed that an unseen, dark third body in the system cannot be extracted from the presently available minima observations. All of these makes very unlikely the presence of a third body with a mass and orbit which would cause a peculiar periastron precession.

First time two-colour light curves were presented by us for BP Vul. The light curve solution - using the Wilson-Devinney Code - yielded very similar results comparing to Devor (2005)'s one and Lacy et al. (2003)'s one. In addition, the $O-C$ analysis in this paper showed that the apsidal motion period in BP Vul is prograde and it has a period of about 365 years - it is in agreement with the value determined with less accuracy from the light curve. The prograde motion means that $\mathrm{BP} \mathrm{Vul}$ is not a representative of problematic cases and it is in agreement with theoretical expectations. Nevertheless we concluded that the negative apsidal motion rate determined by Lacy et al. (2003) is only a consequence of their short observational window. 


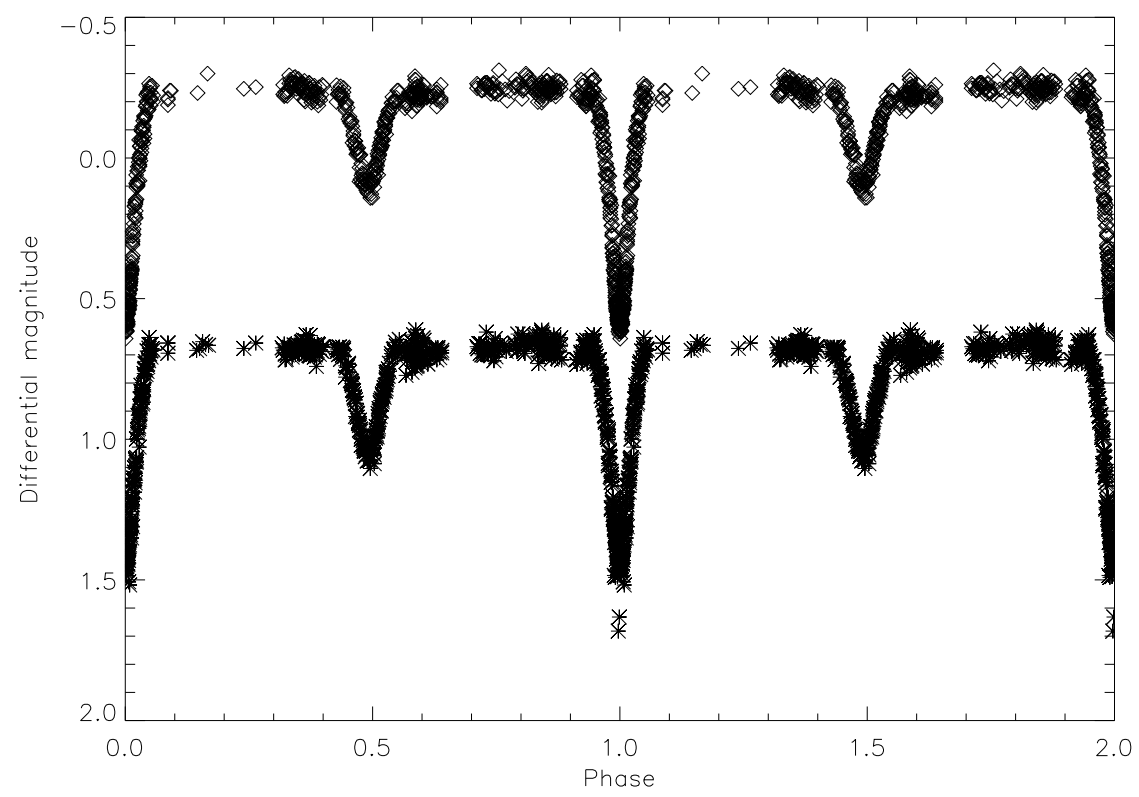

Figure 1: The $B$ (top) and $V$ (bottom) light curves of BP Vul obtained in the years 1959-1963. Bottom is the $\mathrm{B}$ curve while top curve is the $\mathrm{V}$ one which is shifted by 0.3 magnitudes for the sake of clarity.

We also calculated the $k_{2}$ internal structure of $\mathrm{BP} \mathrm{Vul}$ and found it being close to the theoretical value. The slight difference should be refined in the future with a better observed $O-C$ diagram without gaps. Therefore the minima observations of BP Vulpeculae in the future are needed.

The comments on the first version of the manuscript by Drs J. Jurcsik and K. Oláh is acknowledged.

\section{References}

Agerer, F., Dahm, M., Hübscher, J., 2001, IBVS 5017

Borkovits T., Csizmadia Sz., Hegedüs T., Bíró I. B., Sándor Zs., Opitz A., 2002, A\&A 392, 895

Borkovits T., Forgács-Dajka E., Regály Zs., 2007, A\&A 473, 191

Claret, A., 1998, A\&A, 330, 533

Claret, A., 2004, A\&A, 424, 919

Devor, J., 2005, ApJ 628, 411 


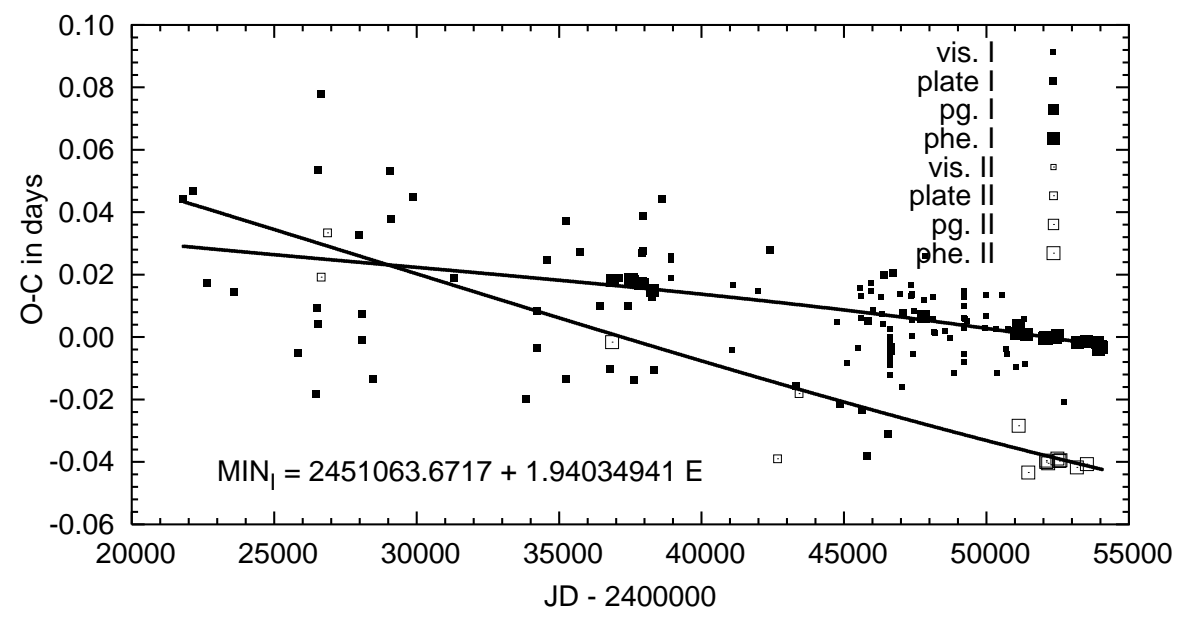

Figure 2: The $O-C$ diagram of BP Vulpeculae. Filled and open squares represent primary and secondary minima, respectively. Lines show the fits to the $O-C$ residuals for the primary and secondary minima, respectively. The weights of the different kind of minima can be found in the text.

Diethelm, R., 2003, IBVS 5438 (BBSAG 129)

Gimenez, A., 1985, ApJ 297, 405

Illés-Almár, E., 1960, ATsir, 210, 21

Huth, H., 1965, IBVS 96

Hübscher, J., Paschke, A., Walter, F., 2006, IBVS 5731 (BAV 178)

Hübscher, J., 2007, IBVS 5802 (BAV 186)

Hübscher, J., Steinbach, H.-M., Walter, F., 2008, IBVS 5830 (BAV 193)

Kwee, K.K., van Woerden, H., 1956, Bull. Astron. Inst. Neth., 12, 327

Lacy, C. H., 1992, AJ 104, 801

Lacy, C. H., 2002, IBVS 5357

Lacy, C. H., 2003, IBVS 5487

Lacy, C. H., 2004, IBVS 5557

Lacy, C. H., 2006, IBVS 5670

Lacy, C. H. S., 2007, IBVS 5764

Lacy, C. H., Marcrum, K., Ibanoglu, C., 1999, IBVS 4737 
Lacy, C. H., Straughn, A., Denger, F., 2002, IBVS 5251

Lacy, C. H., Torres, G., Claret, A., Sabby, J. A., 2003, AJ 126, 1905

Popper, D. M., Etzel, P. B., 1981, AJ 81, 102

Torres, G., Guilbault, P. R., 2003, IBVS 5421

van Hamme, W., 1993, AJ 106, 2096

Wilson, R. E., 1998, Computing Observable Binary Stars (University of Florida)

Zejda, M., 2004, IBVS 5583 